\title{
A huge pleural hydatid cyst with significant shift of mediastinum
}

\author{
Reza Rezaei ${ }^{1}$, Vahid Zehi' ${ }^{2}$, Golnaz Seyedin ${ }^{2}$ \\ ${ }^{1}$ Lung Diseases Research Centre, Mashhad University of Medical Sciences, Mashhad, Iran \\ ${ }^{2}$ Department of General Surgery, Mashhad University of Medical Sciences, Mashhad, Iran
}

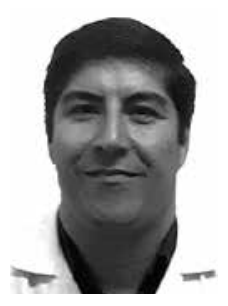

Kardiochir Torakochir Pol 2021; 18 (1): 64-65

Echinococcosis, or hydatid cystic disease, is caused by the larvae of the Echinococcus tapeworm. The annual incidence may be as high as 13 to 27 cases/1,000,000 in certain parts of central Asia. Its incidence in Iran is still high. If they pass the obstacle of the liver, they are arrested in the lung. There is also evidence that the disease can be transmitted through the bronchi. Also, it has been theorized that extra parenchymal and intrathoracic hydatids may develop inside of the chest wall via transmission of the lymphatics of the chest wall [1]. Pleural involvement is rare and usually follows the rupture of a pulmonary or hepatic cyst inside the pleural space causing secondary pleural hy-

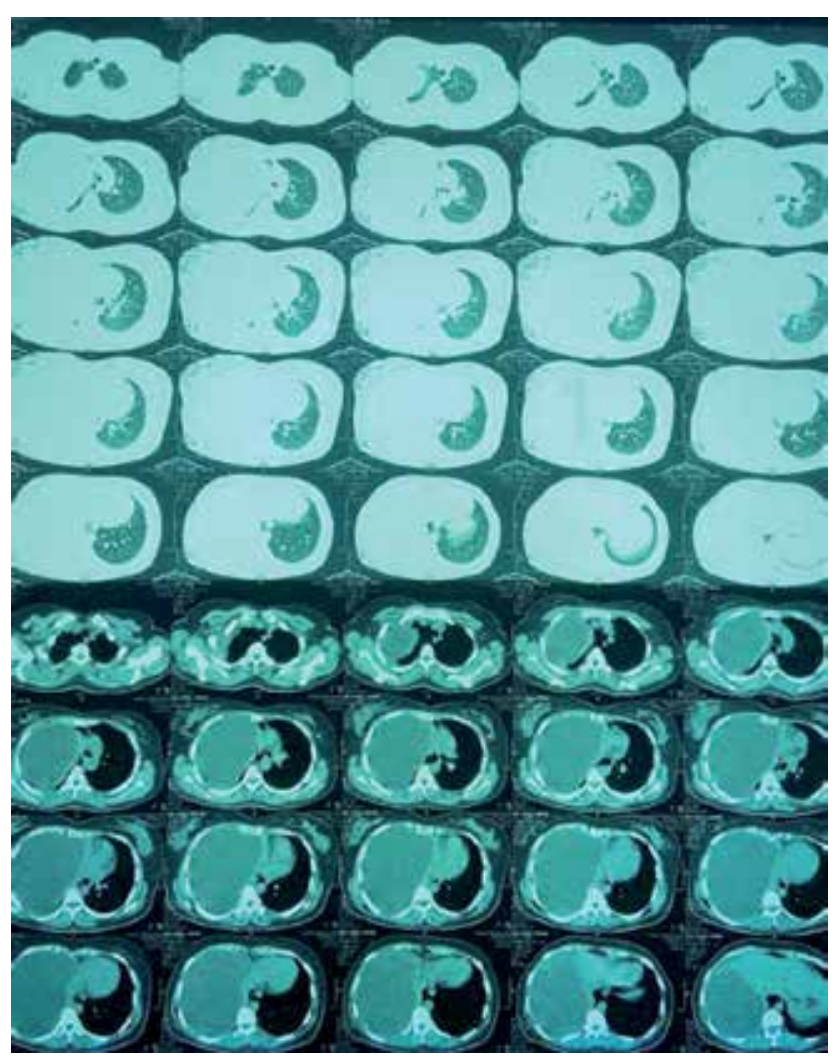

Figure 1. Huge cystic lesion with severe mediastinal shift datidosis. Intrathoracic yet extra pulmonary locations are infrequent, with an occurrence rate of $7.4 \%$ [2]. It may be primarily caused by direct haematological or lymphatic larval seeding or secondarily as a late complication of surgery or chest tube insertion during therapy for thoracic hydatid disease [3].

Herein we present the case of a 33-year-old female, who came to us with the complaint of gradually worsening dyspnoea for the preceding 4 months. Clinical examination revealed decreased breath sounds over the right lung. Computed tomography (CT) scan of the thorax and abdomen showed a huge cyst in the right hemithorax containing many daughter cysts, which led to complete lung collapse and a significant shift of the midline structure with simultaneous liver hydatidosis. Most probably a large liver cyst raised the diaphragm and caused lung collapse and shifted mediastinal structure, so the patient was ready for thoracotomy (Figure 1).

Right posterolateral thoracotomy through the $6^{\text {th }}$ intercostal space was done. Due to the large size of the cyst, and in order to prevent spillage of the cyst, after partial cutting of the intercostal muscles, using a special trocar, some of the contents of the cyst were drained and then a complete incision was made through the entire thickness of the muscles. We encountered a large pleural hydatid cyst that contained a lot of daughter cysts (Figure 2).

By performing the principles of complete evacuation, hydatid cyst drainage was done. After complete evacuation of the daughter cysts, the membrane of the pleural hydatid cyst was carefully removed from the thoracic wall, lung parenchyma, pericardium, and diaphragm. The pulmonary parenchyma was highly intact. The membrane of the cyst had communication with the sub-diaphragmatic area through a very small stem that was about 3-4 mm in length. As a result, the diaphragm was opened via the tract, and numerous liver daughter cysts that soaked in bile and solved by bile were seen. The cysts were removed as much as possible. The remarkable point was that the pleural daughter cysts were healthy and active; however, the liver daughter cysts were destroyed by bile. Then the

Address for correspondence: Golnaz Seyedin, Department of General Surgery, Mashhad University of Medical Sciences, Mashhad, Iran, e-mail: golnazseyedin@yahoo.com

Received: 29.10.2020, accepted: 19.01.2021. 
cavity of the liver hydatid cyst was drained by the insertion of a Petzer drain that came out via the abdominal wall. Then we repaired the diaphragm, and the lung was completely expanded. The wound was closed after placing a chest tube. After surgery, the patient was treated with Albendazole $10 \mathrm{mg} / \mathrm{kg}$ daily, and recurrence did not occur in our follow-up.

The hydatid cyst is a parasitic disease that is still endemic in several parts of the world, especially around the Mediterranean rim. Lung parenchymal tissue elasticity defines the growth rate of the cyst. It has been claimed that cysts grow faster in the lung than in the liver because the lung is softer and more elastic than the liver. Negative intrathoracic pressure may be another reason for faster growth in lung tissue. Intrathoracic extra pulmonary hydatid cysts are rare. Amongst them, 55\% are located in the intrapulmonary fissure, $18 \%$ within the parietal pleura, 14\% in the chest wall, $4.5 \%$ in the mediastinum, and $4.5 \%$ in the diaphragm $[1,3,4]$.

Pleural hydatid cysts can develop chiefly as a result of liver or lung cyst rupture into the pleural space with complications of pneumothorax, pleural effusion, or empyema. In the pleura, cysts sit between the parietal pleura and the endothoracic fascia, and the involvement appears to be systemic or lymphatic. The pleural layers are avascular, and a hydatid cyst may form and grow in this region because the structure of the laminated cyst membrane is permeable to calcium, potassium, chloride, water, and urea. Accordingly, these nutritional substances and others that may be useful to the parasite can traverse the membrane via diffusion $[2,3,4]$.

Imaging is a fundamental element for positive diagnosis. Radiographically, a cyst appears as a smooth, round, dense opacity. The germinative membrane may be visible by CT. Such scans are helpful in showing the water density in intact cysts, but in complex cysts the increased density can be confused with mass lesions [5].

Hydatid cysts can form in different anatomical sites, but extrapulmonary location within the thorax is very rare. This rarity may cause difficulties in diagnosis, followed by the unexpected necessity of wide resections and reconstructive procedures

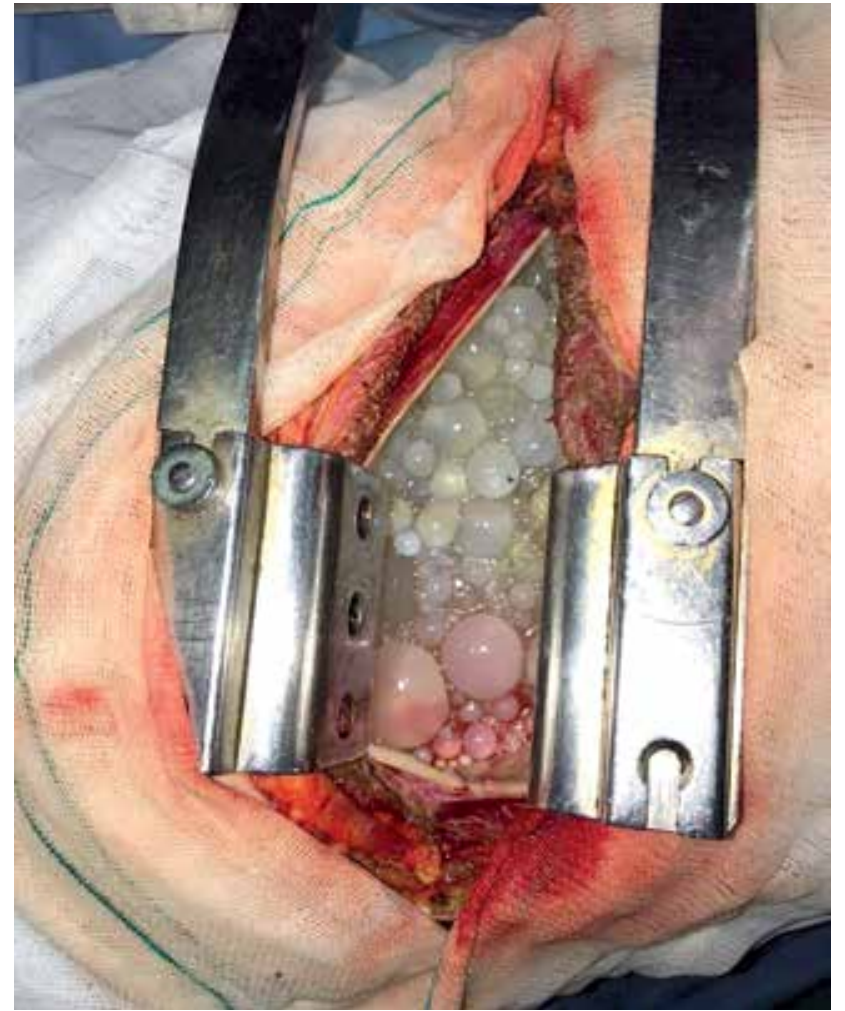

Figure 2. Pleural hydatid cyst with abundant daughter cysts

\section{Disclosure}

The authors report no conflict of interest.

\section{References}

1. Locicero J, Feins R, Colson Y, Rocco G. Shields General Thoracic Surgery. $8^{\text {th }}$ ed. Wolters Kluwer Health, Philadelphia 2018.

2. Teng M, Dawa C, Lakpa P, Tse B. Pulmonary echinococcosis in China. J Thorac Dis 2019; 11: 3146-3155.

3. Gursoy S, Ucvet A, Tozum H, Erbaycu E, Kul C. Primary intrathoracic extra pulmonary hydatid cysts. Tex Heart Inst J 2009; 36: 230-233.

4. Feki W, Ketata W, Bahloul N, Msaad S, Kammoun S. Secondary pleural hydatidosis: complication of intrapulmonary echinococcosis. Lung India 2014; 31: 270-273.

5. Odev K, Bilgin K, Nayman A, Altınok T, Küçükapan A. Imaging of cystic and cyst-like lesions of the mediastinum with pathologic correlation. J Clin Imaging Sci 2012; 2: 33-36. 